\title{
A cost-effective method to immobilize hydrated soft-tissue samples for atomic force microscopy
}

Suchit Sahai ${ }^{1}$, Marysuna Wilkerson ${ }^{1 \dagger}$, Ana Maria Zaske ${ }^{2}$, Scott D. Olson ${ }^{1}$, Charles S. Cox Jr. ${ }^{1}$, and Fabio Triolo ${ }^{1}$

${ }^{1}$ Department of Pediatric Surgery, McGovern Medical School, UTHealth

- The University of Texas Health Science Center at Houston, Houston, TX and ${ }^{2}$ Department of Internal Medicine, Division of Cardiology, UTHealth The University of Texas Health Science Center at Houston, Houston, TX

${ }^{\dagger}$ Current affiliation: UTMB School of Medicine, UTMB Health - The University of Texas Medical Branch at Galveston, Galveston, TX

BioTechniques 61:206-209 (October 2016) doi 10.2144/000114461

Keywords: atomic force microscopy; soft tissue; Wharton's jelly; transglutaminase; polyphenolic proteins; bioadhesives; wet-adhesion

Supplementary material for this article is available at www.BioTechniques.com/article/114461.

Immobilizing hydrated soft tissue specimens for atomic force microscopy (AFM) is a challenge. Here, we describe a simple and very cost-effective immobilization method, based on the use of transglutaminase in an aqueous environment, and successfully apply it to AFM characterization of human native Wharton's Jelly (nWJ), the gelatinous connective tissue matrix of the umbilical cord. A side-byside comparison with a widely used polyphenolic protein-based tissue adhesive (Corning Cell-Tak), which is known to bind strongly to virtually all inorganic and organic surfaces in aqueous environments, shows that both adhesives successfully immobilize nWJ in its physiological hydrated state. The cost of transglutaminase, however, is over 3000-fold lower than that of Cell-Tak, making it a very attractive method for immobilizing soft tissues for AFM characterization.

Biomaterials present exciting strategies for repairing damaged tissues; therefore, understanding the mechanical properties of biomaterials is fundamental to defining critical design considerations for implant success. For over two decades, atomic force microscopy (AFM) has been the gold standard for measuring the mechanical properties of both biomaterials and cells at high spatial resolution and force sensi- tivity within physiologically relevant environments (1-3). However, while determining the mechanical properties of hard scaffolds is relatively straightforward, mechanical testing of soft tissue scaffolds poses significant challenges, the primary of which is sample mounting. A good immobilization technique prevents formation of artifacts, and while many methods (e.g., those based on physical adsorption, covalent binding, air-drying, or use of porous polymer membranes) work well on single-cell layers, soft biological tissues are more technically challenging to mount due to the nature of matrix proteins, fiber orientation, and hydration level (2,4-7). A particularly interesting natural soft biomaterial of great therapeutic potential is Wharton's Jelly, the stem cell-rich connective tissue of the umbilical cord. We used AFM to study its biomechanical properties, of which very little is known despite their importance in defining critical design considerations for efficient tissue engineering applications. With no previously reported AFM studies of this system, the first technical hurdle to overcome was identifying an effective method to immobilize Wharton's Jelly in its native hydrated state (nWJ) to enable AFM analysis. To do so, we explored two different tissue adhesives: (i) Cell-Tak (Corning, New York, NY), which has previously been used for AFM sample preparation $(5,8)$ and is based on polyphenolic proteins extracted from the marine mussel Mytilus edulis; and (ii) transglutaminase (Moo Gloo, Portsmouth, $\mathrm{NH}$ ), also known as "meat glue," an enzyme that catalyzes the formation of protein networks by introducing glutamyl-lysyl isopeptide bonds between target proteins and is commonly used in commercial food processing to bond different proteincontaining foods together $(9,10)$.

Umbilical cords were collected after donor consent at Memorial Hermann Hospital in Houston, TX. Umbilical cords from hepatitis $B$ virus ( $\mathrm{HBV}$ ), hepatitis $\mathrm{C}$ virus (HCV), or HIV positive mothers or newborns were excluded. After harvesting nWJ from the middle portions of the umbilical cords, each $\sim 5 \mathrm{~mm}$ (diameter) $\times \sim 1 \mathrm{~mm}$ (thickness) sample was immobilized on a $1 \mathrm{~cm}^{2}$ area of a $60 \mathrm{~mm}$ diameter, negatively charged polystyrene dish (Cat. \#353002, Corning) pre-coated with 1 of the 2 adhesives. For Cell-Tak we followed the manufacturer's hand-spreading method, spreading $10 \mu$ l of Cell-Tak in 5\% acetic acid manually using a micropipette tip. After the acetic acid evaporated, the dish was washed with ethanol and water. nWJ was placed on the coated surface for 30 min, during which time the strongly hydrophilic nWJ remained fully hydrated, and then was immersed in buffer consisting of $1 \times$ DPBS with calcium and magnesium, $\mathrm{pH}$

\section{METHOD SUMMARY}

This paper describes a simple, very cost effective, and artifact-free method to immobilize hydrated soft tissue samples for atomic force microscopy (AFM) applications by using transglutaminase in a hydrated environment. 
7.0, ionic strength $0.2 \mathrm{M}$ (Cat. \#21-030-CV, Mediatech, Manassas, VA) for analysis. When using transglutaminase, a slurry was prepared according to the manufacturer's instructions by mixing enzyme and deionized water in a 1:4 (w/v) ratio. After letting the slurry set for 10 min on the dish, nWJ was placed on the coated surface for $30 \mathrm{~min}$ and then immersed in buffer solution for analysis. Data were acquired at room temperature using a Bruker BioScope II Controller (Bruker Corporation, Fremont, CA) integrated with a Nikon TE2000-E inverted optical microscope (Nikon Instruments Inc., Melville, NY) and were analyzed using NanoScope software (Bruker). Force curves were registered using Novascan colloidal AFM probes (Ames, IA) consisting of a 5- $\mu \mathrm{m}$ diameter borosilicate glass particle attached to the edge of a silicon nitride $\mathrm{V}$-shaped cantilever with a nominal spring constant of $0.24 \mathrm{~N} / \mathrm{m}$ according to manufacturer's stated values. Indentation curves were captured using 3and 5- $\mu \mathrm{m}$ ramp sizes, corresponding to an indentation depth of $\sim 1 \mu \mathrm{m}$, a scan rate of 0.5 $\mathrm{Hz}$, and a trigger threshold of $12.69 \mathrm{nN}=1$ $\checkmark$ for each experiment. The nWJ was found to behave elastically rather than viscoelastically at this loading rate. Young's modulus (YM) was calculated following the Hertz model (spherical indenter radius $=2.5 \mu \mathrm{m}$ ) with a Poisson's ratio of 0.4 (11). YM calculation analysis was performed using the NanoScope Analysis software.

The mean YM (E) values for nWJ samples immobilized using Cell-Tak and transglutaminase were $2.06 \pm 1.81$ and $2.50 \pm 0.37$ $\mathrm{kPa}$, respectively, consistent with the YM range of some of the body's softest tissues (12). The corresponding figures (Figure 1, A1 and A2; Figure 1, B1 and B2) include graphs of the YM (E) data distribution and the representative AFM force-distance curves, which show the elastic behavior of the tissue sample, indicating the tissue maintained its physiological properties when immobilized with either Cell-Tak or transglutaminase. Overall, the data show that nWJ immobilized using Cell-Tak or transglutaminase exhibits a similar elasticity range, indicating that both adhesives are functionally effective

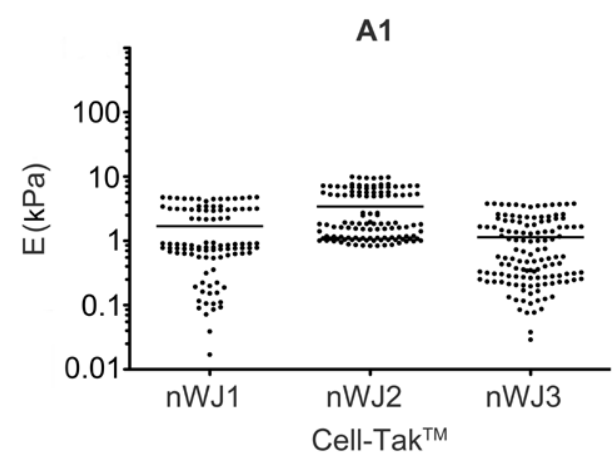

15

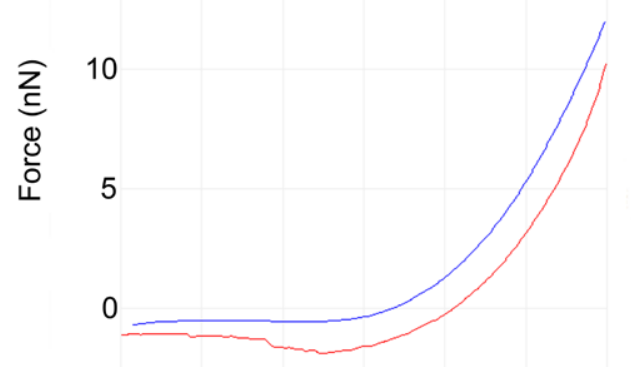

$\begin{array}{lllll}0.5 & 1 & 1.5 & 2 & 2.5\end{array}$

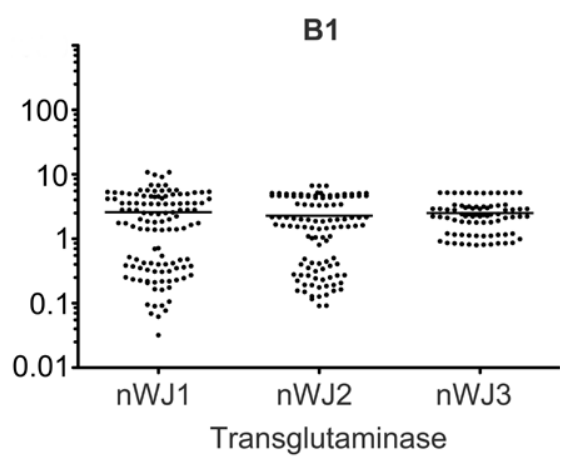

B2
15

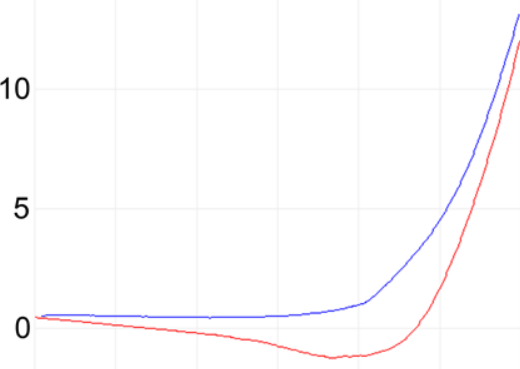

$\begin{array}{llccc}0.5 & 1 & 1.5 & 2 & 2.5 \\ & & Z(\mu \mathrm{m})\end{array}$

Figure 1. Young's Moduli and approach (blue) and retraction (red) force distance curves of human native Wharton's Jelly (nWJ) immobilized using different adhesives. Graphs show the distribution of Young's Moduli (E) in nWJ samples immobilized using Cell-Tak (Panel A1) and transglutaminase (Panel B1) and the corresponding representative atomic force microscopy (AFM) force distance curves (Panels A2 and B2). Seven to eight regions in each sample were randomly selected, and 8-12 data points per region were acquired. Every group of data points represents one region ramped several times. nWJ1, nWJ2, and nWJ3 are samples harvested from three different umbilical cords.

in immobilizing nWJ in its physiological hydrated state; however, their cost-effectiveness is drastically different. Coating a $1 \mathrm{~cm}^{2}$ surface area with Cell-Tak costs \$2 versus $\$ 0.00065$ for transglutaminase (Table 1), the latter being over 3000 times more cost-effective. AFM samples can also be mounted with fibrin sealants $(7,13)$. Although we did not test their performance with nWJ, their average cost per milliliter is 1000-fold higher than that of transglutaminase. The very low cost, coupled with the simplicity and robustness of the sample preparation method, makes the use of transglutaminase an excellent option for immobilizing soft tissues for AFM applications.

Cell-Tak and transglutaminase utilize biological mechanisms of adhesion.

Table 1. Cell-Tak vs. transglutaminase: Comparison of mounted Yissue Young's modulus and adhesive cost.

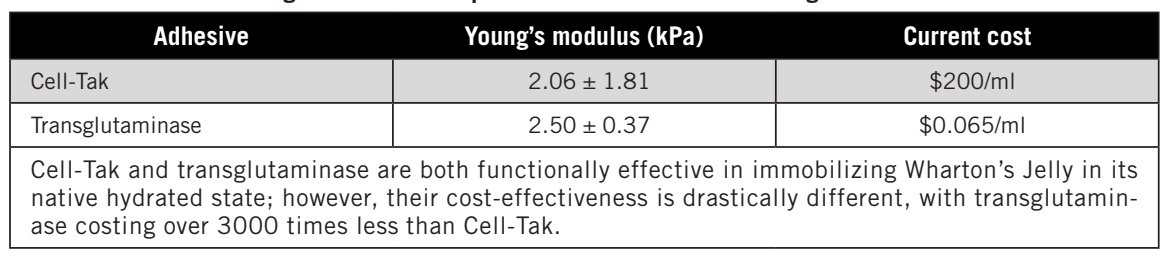

Cell-Tak consists of polyphenolic proteins that bind strongly to virtually all inorganic and organic surfaces in aqueous environments, a property that is believed to be mediated by 3,4-dihydroxy-L-phenylalanine (L-DOPA) (8). Transglutaminase forms glutamyl-lysyl isopeptide bonds between target proteins and likely immobilizes nWJ by cross-linking itself via its reactive lysine residues (10) to glutamine-containing substrates in nWJ.

To our knowledge, this is the first study to use transglutaminase to mount AFM samples and the first to describe a method for immobilizing nWJ, enabling the investigation of its mechanical properties using AFM. Although the effectiveness of our approach could possibly be affected by tissue type (e.g., variability in tissue charge-nWJ is negatively charged in this buffer) and experimental conditions (e.g., buffer strength), it is likely applicable to other soft tissues that need to be studied in the hydrated state, since glutamine is the most abundant naturally occurring nonessential amino acid in human tissues (14). 


\section{Author contributions}

S.S. contributed to experimental design and to the development of the nWJ isolation procedure, prepared the AFM samples, and wrote the manuscript. M.S.W. contributed to the development of the nWJ isolation procedure, prepared the AFM samples, and edited the manuscript. A.M.Z. carried out the AFM experiments. S.D.O. suggested key ideas to immobilize the tissue and edited the manuscript. C.S.C. and F.T. were key contributors to experimental design, coordinated the study, and prepared the manuscript.

\section{Acknowledgments}

This research project has been approved by the Committee for the Protection of Human Subjects at UTHealth (protocol HSC-MS-11-0601). We thank Sufira Kiran for her assistance in consenting and collecting the umbilical cords from the Hospital.

\section{Competing interests}

The authors declare no competing interests.

\section{References}

Wilson L., P. Matsudaira, B. Jena, J.K. Horber. 2002. Atomic Force Microscopy in Cell Biology. Academic Press, San Diego, CA.

2. Kasas, S., N.H. Thomson, B.L. Smith, P.K. Hansma, J. Miklossy, and H.G. Hansma. 1997. Biological applications of the AFM: From single molecules to organs. Int. J. Imaging Syst. Technol. 8:151-161.

3. Kilpatrick, J.I., I. Revenko, and B.J. Rodriguez. 2015. Nanomechanics of cells and biomaterials studied by atomic force microscopy. Adv Healthc Mater. 4:2456-2474.

4. El Kirat, K., I. Burton, V. Dupres, and Y.F. Dufrene. 2005. Sample preparation procedures for biological atomic force microscopy. J. Microsc. 218:199-207.

5. Louise Meyer, R., X. Zhou, L. Tang, A. Arpanaei, P. Kingshott, and F. Besenbacher. 2010. Immobilisation of living bacteria for AFM imaging under physiological conditions. Ultramicroscopy 110:1349-1357.

6. Coburn, J.C. and A. Pandit. 2007. Development of Naturally-Derived Biomaterials and Optimization of Their Biomechanical Properties, p. 1-23. In N. Ashammakhi, R. Reis, and E. Chiellini, Topics in Tissue Engineering. University of Oulu, Helsinki, Finland 13:1-23.

7. Morgan, J.T., V.K. Raghunathan, S.M. Thomasy, C.J. Murphy, and P. Russell. 2014. Robust and artifact-free mounting of tissue samples for atomic force microscopy. Biotechniques 56:40-42.

8. Lee, H., N.F. Scherer, and P.B. Messersmith. 2006. Single-molecule mechanics of mussel adhesion. Proc. Natl. Acad. Sci. USA 103:12999-13003.

9. Martins, I.M., M. Matos, R. Costa, F. Silva, A Pascoal, L.M. Estevinho, and A.B. Choupina. 2014. Transglutaminases: recent achievements and new sources. Appl. Microbiol. Biotechnol. 98:6957-6964.

10. Heck, T., G. Faccio, M. Richter, and L. Thony-Meyer. 2013. Enzyme-catalyzed protein crosslinking. Appl. Microbiol. Biotechnol. 97:461-475.

11. Liu, F. and D.J. Tschumperlin. 2011. Micromechanical characterization of lung tissue using atomic force microscopy. J. Vis. Exp. 54:2911.

12. Cox, T.R. and J.T. Erler. 2011. Remodeling and homeostasis of the extracellular matrix: implications for fibrotic diseases and cancer. Dis Model Mech. 4:165-178.

13. Chaurasia, S.S., R. Champakalakshmi, A. Li, R. Poh, X.W. Tan, R. Lakshminarayanan, C.T. Lim, D.T. Tan, and J.S. Mehta. 2012. Effect of fibrin glue on the biomechanical properties of human Descemet's membrane. PLoS ONE 7:e37456.

14. Miller, A.L. 1999. Therapeutic considerations of L-glutamine: a review of the literature. Altern. Med. Rev. 4:239-248.

Received 23 March 2016; accepted 08 July 2016.

Address correspondence to: Fabio Triolo, UTHealth, 1941 East Rd., BBSB 6106, Houston, TX, 77054. Email: fabio.triolo@uth.tmc.edu

To purchase reprints of this article, contact: biotechniques@fosterprinting.com

\section{Introducing the New Programmable Nanoject III}

Proven Hydraulic Technology Ensures Consistent Injection Volumes

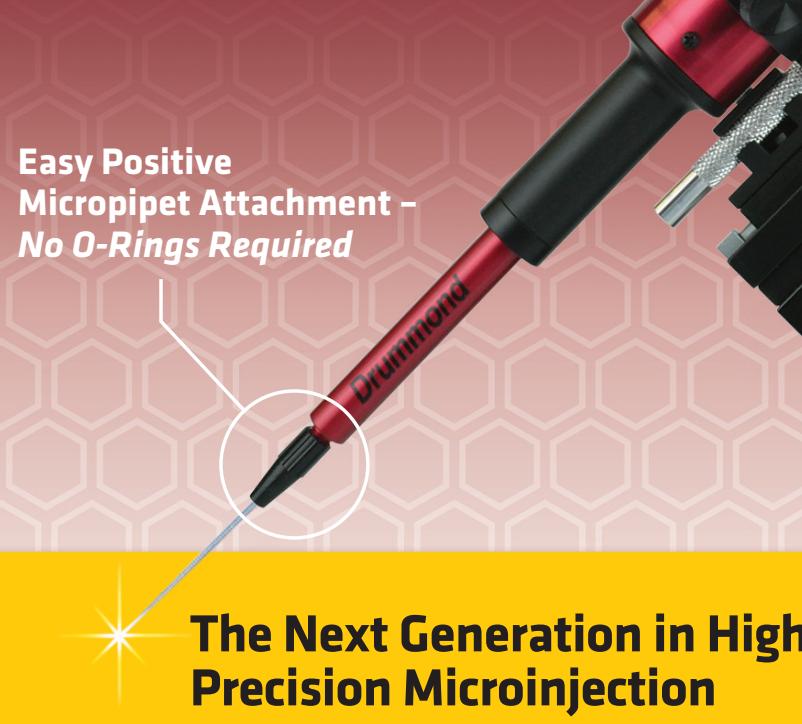

The Next Generation in High Precision Microinjection
User Friendly Touchscreen Operation Potential Applications Expanded 